\title{
Comparison of Efficacy and Safety Profile of Sodium-Glucose Cotransporter-2 Inhibitors as Add-On Therapy in Patients With Type 2 Diabetes
}

Mazhar Hussain ${ }^{1}$, Asim Elahi ${ }^{2}$, Javed Iqbal ${ }^{3}$, Muhammad Bilal Ghafoor ${ }^{4}$, Habib Rehman ${ }^{3}$, Shoaib Akhtar 3

1. Pharmacology, Sheikh Zayed Medical College and Hospital, Rahim Yar Khan, PAK 2. Internal Medicine, Pikeville Medical Center, Pikeville, USA 3. Medicine, Sheikh Zayed Medical College and Hospital, Rahim Yar Khan, PAK 4. Pathology, Sheikh Zayed Medical College and Hospital, Rahim Yar Khan, PAK

Corresponding author: Mazhar Hussain, mazharhussain214@gmail.com

\section{Abstract}

Background

Type 2 diabetes is a chronic metabolic disorder that is escalating at an alarming rate worldwide. Sodiumglucose cotransporter-2 (SGLT-2) inhibitors are recent oral antihyperglycemic drugs (OADs) with a unique mechanism of action.

Objectives

This study aimed compared the efficacy and safety profiles of two SGLT-2 inhibitors, empagliflozin and dapagliflozin, in patients with type 2 diabetes as add-on therapy to traditional first-line OADs.

Methods

We conducted a randomized controlled trial comparing empagliflozin and dapagliflozin in patients with type 2 diabetes. Patients were included in the study if they had type 2 diabetes with inadequate glycemic control, defined as glycated hemoglobin (HbA1c) of $7.5 \%$ to $11.0 \%$, treated with conventional first-line OADs. Study participants were randomly assigned into two groups. Group A patients received oral empagliflozin, 10 to 25 $\mathrm{mg}$, and Group B patients received oral dapagliflozin, 5 to $10 \mathrm{mg}$, for 12 weeks. The primary endpoint was the efficacy profile for each SGLT-2 agent in terms of body weight changes, body mass index (BMI), fasting blood glucose (FBG), and HbA1c. The secondary endpoint was to determine the safety and tolerability profiles of each SGLT-2 agent.

Results

Review began 03/17/2021 Review ended 03/27/2021 Published 04/03/2021

\section{() Copyright 2021}

Hussain et al. This is an open access article distributed under the terms of the Creative Commons Attribution License CC-BY 4.0., which permits unrestricted use, distribution, and reproduction in any medium, provided the original author and source are credited.
After 12 weeks of treatment, the mean body weight was reduced significantly in both groups from baseline (empagliflozin: $-3.2 \mathrm{~kg} \pm 5.5 \mathrm{~kg}, \mathrm{p}=0.003$; dapagliflozin $-2.1 \mathrm{~kg} \pm 4.6 \mathrm{~kg}, \mathrm{p}=0.008$ ). However, the mean body weight reduction between groups was not statistically significant $(\mathrm{p}=0.078)$. BMI was significantly reduced in both groups (empagliflozin from $28.5 \pm 4.9 \mathrm{~kg} / \mathrm{m}^{2}$ to $25.8 \pm 5.2 \mathrm{~kg} / \mathrm{m}^{2}, \mathrm{p}=0.002$; dapagliflozin from $29 \pm$ $5.2 \mathrm{~kg} / \mathrm{m}^{2}$ to $27.7 \pm 4.8 \mathrm{~kg} / \mathrm{m}^{2}, \mathrm{p}=0.003$ ). However, the patients who received empagliflozin experienced a significantly greater reduction in BMI than patients who received dapagliflozin $(\mathrm{p}=0.007)$.

The mean FBG was also reduced in both study groups (empagliflozin: $-88.5 \mathrm{mg} / \mathrm{dL} \pm 39.7 \mathrm{mg} / \mathrm{dl}, \mathrm{p}=0.003$; dapagliflozin: $-59.8 \mathrm{mg} / \mathrm{dL} \pm 48.5 \mathrm{mg} / \mathrm{dL} ; \mathrm{p}=0.007$ ). However, the patients who received empagliflozin experienced a significantly greater reduction in mean FBG than patients who received dapagliflozin $(\mathrm{p}=$ 0.001 ). HbA1c was also significantly reduced in both groups (empagliflozin: $-2.1 \% \pm 1.1 \%, \mathrm{p}=0.002$; dapagliflozin: $-1.4 \% \pm 0.9 \% ; \mathrm{p}=0.004$ ). However, patients who received empagliflozin experienced a significantly greater reduction in HbA1c than patients who received dapagliflozin $(p=0.001)$.

The tolerability profiles of both SGLT-2 agents were quite good, and no major adverse effects were reported in the study groups. Urinary infection occurred more often in patients who received dapagliflozin (9.3\%) than in patients who received empagliflozin $(4.5 \% ; \mathrm{p}=0.002)$. Patients in the dapagliflozin group also had a higher incidence of genital infections $(7.3 \%)$ than those in the empagliflozin group $(3.8 \%$; $\mathrm{p}=0.001)$.

Conclusion

Both empagliflozin and dapagliflozin demonstrated excellent efficacy and safety profiles in our study. These agents should be considered as add-on therapy in patients with type 2 diabetes taking conventional first-line OADs. 
Categories: Endocrinology/Diabetes/Metabolism, Emergency Medicine, Family/General Practice Keywords: efficacy, safety profile, adverse effects, sodium-glucose cotransporter-2 (sglt-2) inhibitors, glycated hemoglobin (hba1c), body mass index (bmi)

\section{Introduction}

Type 2 diabetes is a chronic metabolic disorder with an escalating incidence worldwide, found in nearly one in 11 people [1]. The number of diabetes cases worldwide is expected to rise from 450 million to 642 million in 20 years. Pakistan has the fourth-most diabetes cases globally, and in 2019, 19.4 million people in Pakistan had diabetes; the number of cases is projected to reach 26.2 million in 2030 and 37.1 million in 2045 [2]. This level of prevalence of diabetes will add significant morbidity and mortality and pose an enormous economic burden on national resources [1-2].

The treatments available for patients with diabetes have seen significant advancements. Oral antihyperglycemic drugs (OADs) are usually first-line therapies and lifestyle changes for patients with type 2 diabetes. There are currently seven first-line OADs, with several more in the development and the approval stages. The current class of OADs consists of biguanides, sulphonylurea, alpha glycosidase inhibitors, thiazolidinediones, glucagon-like peptide-1 (GLP-1) receptor agonists, dipeptidyl peptidase-4 (DPP-4), and sodium-glucose cotransporter-2 (SGLT-2) inhibitors. The antihyperglycemic effects of these drugs are mediated through various mechanisms [3].

SGLT-2 inhibitors are the latest OADs with a unique mechanism of action. The insulin-independent antihyperglycemic effect of SGLT-2 inhibitors is mediated by suppressing the glucose reabsorption in renal tubules, facilitating its excretion in urine. SGLT-2 inhibitors are optimal in this scenario. Given that approximately $90 \%$ of the filtered load of glucose is reabsorbed in the kidney's proximal convoluted tubule, SGLT-2 inhibitors are an innovative approach to reducing glycemia. The United States (US) Food and Drug Association (FDA) has approved three drugs in this class: canagliflozin, dapagliflozin, and empagliflozin [45].

SGLT-2 inhibitors have excellent efficacy, safety, and tolerability profiles without significant risk of hypoglycemia [6]. Beyond improving glycemic control, SGLT-2 inhibitors offer pleiotropic effects on body weight, blood pressure, hyperuricemia, dyslipidemia, and fatty liver disease [7]. Clinical trials conducted in the US and Europe have shown favorable SGLT-2 safety in cardiovascular and kidney disease [8-9]. However, in Asia, data are limited, and in Pakistan, no study has been conducted to assess the cardiovascular safety profile of SGLT-2 inhibitors. SGLT-2 inhibitors are generally reserved as a second or third-line antihyperglycemic drug in the treatment of type 2 diabetes, but they can also be used as monotherapy when metformin is contraindicated [10].

The goal of this study was to assess the 12-week safety and tolerability profile of dapagliflozin and empagliflozin as add-on therapy in patients with type 2 diabetes currently being treated with conventional first-line OADs.

\section{Materials And Methods}

We conducted a 12-week randomized, controlled trial at five private clinics and the diabetes clinic of Sheikh Zayed Hospital. A total of 615 patients were recruited from these clinical settings, and 342 patients met the inclusion criteria and were enrolled in the study. To be included in the study, patients had to have type 2 diabetes with inadequate glycemic control defined as glycated hemoglobin (HbA1c) of 7.5\% to 11\% treated with different first-line OAD combinations, such as metformin, pioglitazone, glibenclamide, glimepiride, sitagliptin, and vildagliptin. The study excluded patients with a history of type 1 diabetes, ketoacidosis, significant hepatic disease, cardiovascular disease, unstable/rapidly progressing renal disease, malignancy, pancreatic disorders, genitourinary infection, and steroid use. All study participants provided informed written consent for inclusion in the study. The study protocol was approved by the Institutional Review Board of Sheikh Zayed Medical College and Hospital.

The study population was divided into two groups via random sampling. Group A patients were given oncedaily oral empagliflozin, $10 \mathrm{mg}$ to $25 \mathrm{mg}$, and patients in Group B were given once-daily dapagliflozin, $5 \mathrm{mg}$ to $10 \mathrm{mg}$, as an add-on therapy for 12 weeks. The doses of SGLT-2 inhibitors, dipeptidyl peptidase-4 (DPP-4) inhibitors, thiazolidinediones, and metformin were maintained during the study, while sulphonylurea doses were reduced if patients developed hypoglycemic episodes.

The study's primary endpoint assessed each drug's efficacy by measuring body weight changes, body mass index (BMI), fasting blood glucose (FBG), and HbA1c from baseline. The secondary endpoint was determining each drug's safety and tolerability profiles by assessing adverse effects from baseline. All the parameters were measured before starting SGLT-2 inhibitor therapy and at the end of the study. FBG was analyzed via the glucose oxidase peroxidase method. HbA1c was measured by liquid chromatography, while fasting serum lipid profile was measured by the enzymatic endpoint method. 


\section{Cureus}

\section{Data analysis}

Data were analyzed using the Statistical Package for Social Sciences (SPSS) for Windows, version 16.0 (SPSS, Inc., Chicago, IL). The values of numeric data were expressed as mean \pm standard deviation (SD). The frequency data were expressed as a percentage. The difference in the primary endpoint from baseline was analyzed using the paired t-test, while the difference in secondary endpoints from baseline was determined via the Chi-square test. We considered $\mathrm{p}<0.05$ as statistically significant and $\mathrm{p}<0.01$ as highly significant.

\section{Results}

A total of 615 patients with type 2 diabetes were recruited, of whom 420 were considered for the study. Seventy-eight patients were excluded, leaving a total of 342 patients included in the study, randomized into two groups. Sixteen patients in the empagliflozin group and 21 patients in the dapagliflozin group were dropped out. One hundred fifty-five patients received empagliflozin, and 150 patients received dapagliflozin (Figure 1).

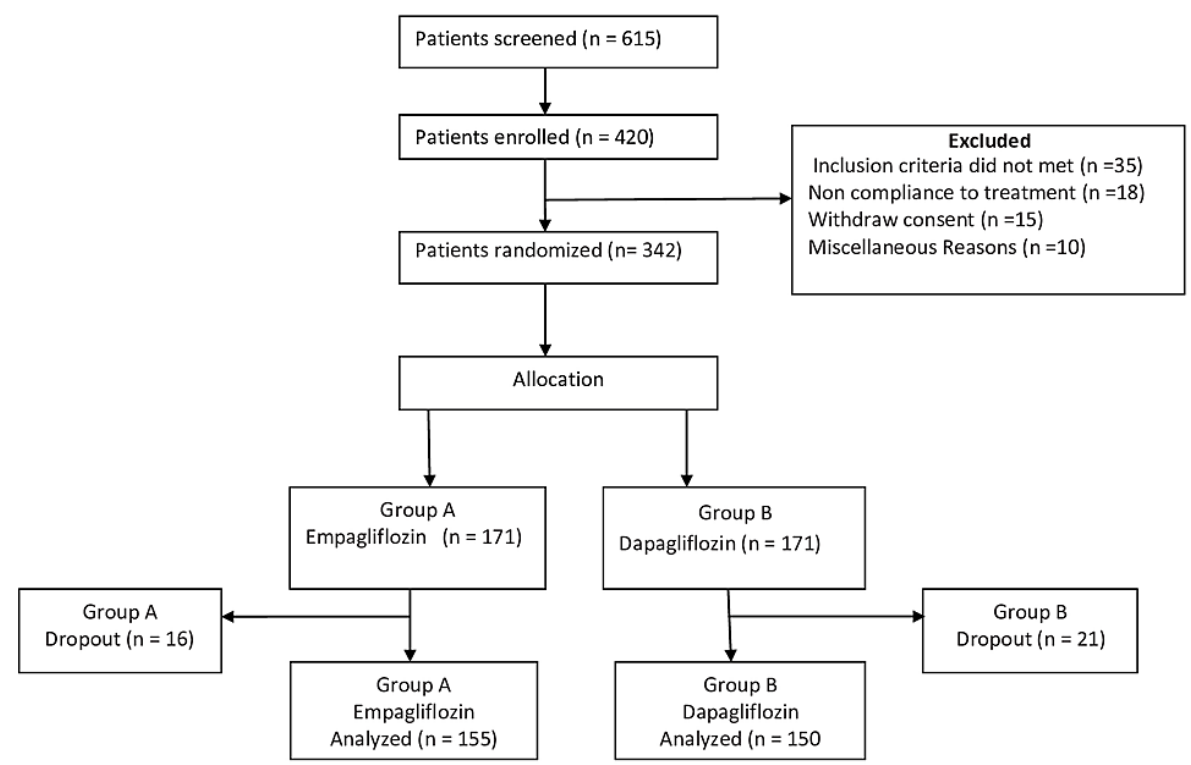

FIGURE 1: Study Flow Chart

There was no difference in the baseline demographic characteristics and clinical study parameters in both study groups at the start of the study. The number of patients taking other OADs is presented in Table 1 . 


\section{Cureus}

\begin{tabular}{|c|c|c|c|}
\hline Patient Characteristics & Empagliflozin ( $n=155)$ & Dapagliflozin $(n=150)$ & P-value \\
\hline Age (years) & $52.5 \pm 15.6$ & $49.4 \pm 18.2$ & 0.63 \\
\hline Gender (M/F) & 109 (70\%)/46 (30\%) & 100 (67\%)/50 (33\%) & 0.32 \\
\hline Body weight (kg) & $93.5 \pm 18.4$ & $95.6 \pm 21$ & 0.42 \\
\hline BMI $\left(\mathrm{kg} / \mathrm{m}^{2}\right)$ & $28.5 \pm 4.9$ & $29 \pm 5.2$ & 0.96 \\
\hline Diabetes duration (years) & $9.2 \pm 3.8$ & $8.9 \pm 5.6$ & 0.72 \\
\hline \multicolumn{4}{|l|}{ Clinical Parameters (mean \pm SD) } \\
\hline FBG (mg/dL) & $188 \pm 36.5$ & $195 \pm 42$ & 0.81 \\
\hline HbA1c (\%) & $10.1 \pm 3.4$ & $9.5 \pm 2.8$ & 0.36 \\
\hline Total Cholesterol (mg/dL) & $164 \pm 32.5$ & $142 \pm 26.5$ & 0.52 \\
\hline Triglycerides (mg/dL) & $187 \pm 29.7$ & $190 \pm 35.6$ & 0.71 \\
\hline LDL Cholesterol (mg/dL) & $98 \pm 32$ & $108 \pm 18.5$ & 0.42 \\
\hline HDL Cholesterol (mg/dL) & $40.5 \pm 8.6$ & $43 \pm 7.8$ & 0.64 \\
\hline \multicolumn{4}{|l|}{ Concomitant OAD (N (\%)) } \\
\hline Biguanides & $21(13.5 \%)$ & $22(14.6 \%)$ & 0.42 \\
\hline Thiazolidinediones & $3(2 \%)$ & $5(3.3 \%)$ & 0.71 \\
\hline DPP-4 Inhibitors & $10(6.4 \%)$ & $12(8 \%)$ & 0.32 \\
\hline Sulphonylurea & $16(10.3 \%)$ & $11(7.3 \%)$ & 0.66 \\
\hline Sulphonylurea + Biguanides & $38(24.5 \%)$ & 42 (28\%) & 0.28 \\
\hline Sulphonylurea + Thiazolidinediones & $10(6.4 \%)$ & $9(6 \%)$ & 0.39 \\
\hline DPP-4 Inhibitors + Biguanides & $40(25.8 \%)$ & 35 (23.3\%) & 0.64 \\
\hline Thiazolidinediones + Biguanides & $17(11 \%)$ & $14(9.3 \%)$ & 0.34 \\
\hline
\end{tabular}

\section{TABLE 1: Baseline Characteristics, Clinical Parameters, and Concomitant Therapies}

BMI: body mass index; DPP-4: dipeptidyl peptidase-4; FBG: fasting blood glucose; HbA1c: glycated hemoglobin; HDL: high-density lipoprotein; LDL: low-density lipoprotein; OAD: oral antihyperglycemic drug; SD: standard deviation

After 12 weeks of treatment, the mean body weight was reduced significantly in both groups. Patients receiving empagliflozin had a mean body weight reduction from baseline of $3.2 \mathrm{~kg} \pm 5.5 \mathrm{~kg}(\mathrm{p}=0.003)$, and patients receiving dapagliflozin had a mean body weight reduction of $2.1 \mathrm{~kg} \pm 4.6 \mathrm{~kg}(\mathrm{p}=0.008)$. The difference in mean body weight between patients receiving empagliflozin and patients receiving dapagliflozin at the end of the study was not statistically significant $(p=0.078)$. BMI was significantly reduced in both groups (empagliflozin from $28.5 \pm 4.9 \mathrm{~kg} / \mathrm{m}^{2}$ to $25.8 \pm 5.2 \mathrm{~kg} / \mathrm{m}^{2}, \mathrm{p}=0.002$; dapagliflozin from $29 \pm 5.2 \mathrm{~kg} / \mathrm{m}^{2}$ to $\left.27.7 \pm 4.8 \mathrm{~kg} / \mathrm{m}^{2}, \mathrm{p}=0.003\right)$. However, the patients who received empagliflozin experienced a significantly greater reduction in BMI than patients who received dapagliflozin $(p=0.007)$.

Similarly, the mean FBG was significantly reduced in both study groups. Patients receiving empagliflozin had a mean FBG reduction of $88.5 \mathrm{mg} / \mathrm{dL} \pm 39.7 \mathrm{mg} / \mathrm{dL}$ ( $\mathrm{p}=0.003$ ). Patients receiving dapagliflozin had a mean FBG reduction of $59.8 \mathrm{mg} / \mathrm{dL} \pm 48.5 \mathrm{mg} / \mathrm{dL}(\mathrm{p}=0.007)$. However, patients who received empagliflozin experienced a significantly greater reduction in mean FBG than patients who received dapagliflozin $(\mathrm{p}=$ 0.001 ) after 12 weeks of treatment.

The mean HbA1c was also significantly reduced in both groups. Patients who received empagliflozin had a mean $\mathrm{HbA1c}$ reduction by $2.1 \% \pm 1.1 \%(\mathrm{p}=0.002)$, and patients who received dapagliflozin had a mean HbA1c reduction by $1.4 \% \pm 0.9 \% \mathrm{p}=0.004)$. Patients who received empagliflozin experienced a significantly greater reduction in the mean HbA1c than patients who received dapagliflozin $(p=0.001)$ after 12 weeks of treatment (Table 2). 


\section{Cureus}

\begin{tabular}{|c|c|c|c|c|c|c|c|c|}
\hline \multicolumn{4}{|c|}{ Group A Empagliflozin ( $n=128)$} & \multicolumn{4}{|c|}{ Group B Dapagliflozin $(n=127)$} & \multirow{2}{*}{ P-valueł } \\
\hline Parameters & 0 weeks & 12 weeks & P-value ${ }^{\prime}$ & Parameters & 0 weeks & 12 weeks & P-value & \\
\hline Body weight (kg) & $93.5 \pm 18.4$ & $89.5 \pm 16.5$ & 0.003 & Body weight (kg) & $95.6 \pm 21$ & $93.4 \pm 17$ & 0.008 & 0.078 \\
\hline BMI $\left(\mathrm{kg} / \mathrm{m}^{2}\right)$ & $28.5 \pm 4.9$ & $25.8 \pm 5.2$ & 0.002 & BMI $\left(\mathrm{kg} / \mathrm{m}^{2}\right)$ & $29 \pm 5.2$ & $27.7 \pm 4.8$ & 0.003 & 0.007 \\
\hline FBG (mg/dL) & $188 \pm 36.5$ & $132.8 \pm 48.5$ & 0.003 & FBG (mg/dL) & $195 \pm 42$ & $151 \pm 38.5$ & 0.007 & 0.001 \\
\hline HbA1c (\%) & $10.1 \pm 3.4$ & $7.5 \pm 2.2$ & 0.002 & HDATC (\%) & $9.5 \pm 2.8$ & $8.2 \pm 3.2$ & 0.004 & 0.001 \\
\hline
\end{tabular}

\section{TABLE 2: Changes From Baseline in Both Study Groups}

† Differences within groups measured at baseline and week 12

‡ Differences within the group measured at baseline and week 12

BMI: body mass index; FBG: fasting blood glucose; HbA1c: glycated hemoglobin

Both drugs' tolerability profiles were quite good, and no major adverse effects were reported in either study group. However, minor adverse effects were observed in both study groups (Table 4).

\begin{tabular}{|c|c|c|c|}
\hline Adverse Effects & Group A Empagliflozin $(n=155)$ & Group B Dapagliflozin $(n=150)$ & P-value* \\
\hline Deaths & 0 & 0 & \\
\hline Adverse effect leading to discontinuation & $5(3.2 \%)$ & $8(5.3 \%)$ & 0.66 \\
\hline Documented hypoglycemia (glucose $<70 \mathrm{mg} / \mathrm{dL}$ ) & $4(2.6 \%)$ & $6(4 \%)$ & 0.48 \\
\hline Hypersensitivity & $2(1.3 \%)$ & $3(2 \%)$ & 0.36 \\
\hline Hypotension/dehydration/hypovolemia & $3(2.0 \%)$ & $4(2.6 \%)$ & 0.25 \\
\hline Frequency/nocturia (three times/night) & $10(6.4 \%)$ & $13(8.6 \%)$ & 0.32 \\
\hline Urinary tract infection & $7(4.5 \%)$ & $14(9.3 \%)$ & 0.002 \\
\hline Genital infection & $6(3.8 \%)$ & $11(7.3 \%)$ & 0.001 \\
\hline Total adverse effects & $37(23.8)$ & $59(39.3)$ & 0.001 \\
\hline
\end{tabular}

\section{TABLE 3: Adverse Effects}

Urinary infections occurred more often in patients who received dapagliflozin (9.3\%) than in patients who received empagliflozin $(4.5 \% ; \mathrm{p}=0.002)$. Patients in the dapagliflozin group also had a higher incidence of genital infections $(7.3 \%)$ than those in the empagliflozin group $(3.8 \%$; $p=0.001)$.

\section{Discussion}

The present study was conducted to determine the efficacy and safety profiles of empagliflozin compared with dapagliflozin as an add-on therapy in patients with type 2 diabetes experiencing inadequate glycemic control with conventional first-line OADs. Both empagliflozin and dapagliflozin have excellent efficacy and safety profiles. However, empagliflozin caused a more significant improvement in mean body weight, FBG, and $\mathrm{HbA1c}$ with fewer adverse effects than dapagliflozin.

The pharmacologic management of diabetes changes over time due to its progressive nature. This makes achieving optimal glycemic control with monotherapy a challenge and eventually causes patients to need two to four OADs as combination therapy, as recommended by both the American Diabetes Association (ADA) and the European Association for the Study of Diabetes (EASD) [11].

In our study, both empagliflozin and dapagliflozin significantly reduced body weight and HbA1c. These results support the findings reported in another study conducted in two different cohorts at 12 and 24 weeks 
where empagliflozin produced similar reductions in body weight and HbA1c [12]. Similarly, another clinical study reported that dapagliflozin reduced HbA1c and body weight over 152 weeks in patients with inadequate glycemic control via metformin [13]. Both studies compared the SGLT-2 inhibitor against a placebo control group, while our study compared the outcomes of two SGLT-inhibitors against each other.

Our results were similar to those of a 52-week study by Ku et al., who reported that empagliflozin reduced body weight, blood glucose levels, and HbA1c to a greater degree than dapagliflozin [14]. Ku et al. also reported that empagliflozin improved cardiometabolic risk factors more significantly than dapagliflozin and had a low incidence of genitourinary infection.

A review regarding the efficacy, safety, and tolerability of different SGLT-2 inhibitors demonstrated that empagliflozin is one of the safer choices and can be prescribed in patients with type 2 diabetes with renal impairment (a parameter that our study did not explore) [15].

A study conducted in the United Arab Emirates reported that canagliflozin (300 mg), in combination with metformin for 26 weeks, provided a greater reduction in HbA1c (-0.79\%) than empagliflozin $25 \mathrm{mg}(-0.64 \%)$ and dapagliflozin $10 \mathrm{mg}(-0.41 \%)$ [16]. A study conducted in China reported that empagliflozin and dapagliflozin reduced body weight, FBG, and $\mathrm{HbA1c}$ for patients with type 2 diabetes, similar to our results [17]. Also, both drugs ameliorated hepatic dysfunction and improved insulin resistance over six months [17].

The efficacy, safety, and tolerability profile of empagliflozin and dapagliflozin as an add-on therapy were investigated in various clinical studies that yielded similar results to our study [18-26]. Both drugs reduced body weight and provided excellent glycemic control with no risk of severe hypoglycemia. Moreover, the risk of urogenital infection varied from $1 \%$ to $9 \%$, which is similar to the risk we found.

The risk of atherosclerotic disease is very high in patients with type 2 diabetes. Treatment with dapagliflozin provides cardiovascular safety with a low rate of cardiovascular death and hospitalization due to heart failure [27]. Empagliflozin also reduces cardiovascular events and delays kidney disease progression in patients with type 2 diabetes with cardiovascular comorbidities. A systematic review and meta-analysis of 27 studies also showed that SGLT-2 inhibitors reduced the risk of renal and cardiovascular disease impairment in patients with chronic kidney disease and patients with diabetes [28].

\section{Conclusions}

Both empagliflozin and dapagliflozin have excellent efficacy, safety, and tolerability profiles. They can be safely used as an add-on therapy to conventional OADs in patients with type 2 diabetes. There is a further need to explore the efficacy and safety of SGLT-2 inhibitors in diabetic patients with cardiovascular disease and renal impairment. Moreover, a study with a larger sample size and longer duration is warranted to confirm the safety and tolerability profile.

\section{Additional Information \\ Disclosures}

Human subjects: Consent was obtained or waived by all participants in this study. Institutional Review Board, Sheikh Zayed Medical College and Hospital issued approval 26/IRB/SZMC/SZH. Animal subjects: All authors have confirmed that this study did not involve animal subjects or tissue. Conflicts of interest: In compliance with the ICMJE uniform disclosure form, all authors declare the following: Payment/services info: All authors have declared that no financial support was received from any organization for the submitted work. Financial relationships: All authors have declared that they have no financial relationships at present or within the previous three years with any organizations that might have an interest in the submitted work. Other relationships: All authors have declared that there are no other relationships or activities that could appear to have influenced the submitted work.

\section{Acknowledgements}

We appreciate the cooperation of Dr. Muhammad Bilal Ghafoor and his laboratory staff.

\section{References}

1. Zheng Y, Ley SH, Hu FB: Global aetiology and epidemiology of type 2 diabetes mellitus and its complications. Nat Rev Endocrinol. 2018, 14:88-98. 10.1038/nrendo.2017.151

2. Saeedi P, Petersohn I, Salpea P, et al.: Global and regional diabetes prevalence estimates for 2019 and projections for 2030 and 2045: results from the International Diabetes Federation Diabetes Atlas. Diabetes Res Clin Prac. 2019, 157:107843. 10.1016/j.diabres.2019.107843

3. Chikara G, Sharma PK, Dwivedi P, Charan J, Ambwani S, Singh S: A narrative review of potential future antidiabetic drugs: should we expect more?. Indian J Clin Biochem. 2018, 33:121-131. 10.1007/s12291-0170668-z

4. Misra M: SGLT2 inhibitors: a promising new therapeutic option for treatment of type 2 diabetes mellitus . J Pharm Pharmacol. 2013, 65:317-327. 10.1111/j.2042-7158.2012.01574.x 
5. Qaseem A, Barry MJ, Humphrey LL, Forciea MA: Oral pharmacologic treatment of type 2 diabetes mellitus: a clinical practice guideline update from the American College of Physicians. Ann Intern Med. 2017, 166:279290. 10.7326/M16-1860

6. Donnan JR, Grandy CA, Chibrikov E, et al.: Comparative safety of the sodium glucose co-transporter 2 (SGLT2) inhibitors: a systematic review and meta-analysis. BMJ Open. 2019, 9:e022577. 10.1136/bmjopen2018-022577

7. Kalra S: Sodium glucose co-transporter-2 (SGLT2) inhibitors: a review of their basic and clinical pharmacology. Diabetes Ther. 2014, 5:355-366. 10.1007/s13300-014-0089-4

8. Satoh H: Pleiotropic effects of SGLT2 inhibitors beyond the effect on glycemic control . Diabetol Int. 2018, 9:212-214. 10.1007/s13340-018-0367-X

9. Garofalo C, Borrelli S, Liberti ME, et al.: SGLT2 inhibitors: nephroprotective efficacy and side effects . Medicina (Kaunas). 2019, 55:268. 10.3390/medicina55060268

10. American Diabetes Association: 9. Pharmacologic approaches to glycemic treatment: standards of medical care in diabetes-2019. Diabetes Care. 2019, 42:S90-S102. 10.2337/dc19-S009

11. Inzucchi SE, Bergenstal RM, Buse JB, et al.: Management of hyperglycaemia in type 2 diabetes: a patientcentered approach. Position statement of the American Diabetes Association (ADA) and the European Association for the Study of Diabetes (EASD). Diabetologia. 2012, 55:1577-1596. 10.1007/s00125-012-25340

12. Neeland IJ, McGuire DK, Chilton R, et al.: Empagliflozin reduces body weight and indices of adipose distribution in patients with type 2 diabetes mellitus. Diab Vasc Dis Res. 2016, 13:119-126. 10.1177/1479164115616901

13. Bolinder J, Ljunggren Ö, Johansson L, et al.: Dapagliflozin maintains glycaemic control while reducing weight and body fat mass over 2 years in patients with type 2 diabetes mellitus inadequately controlled on metformin. Diabetes Obes Metab. 2014, 16:159-169. 10.1111/dom.12189

14. Ku EJ, Lee DH, Jeon HJ, Oh TK: Empagliflozin versus dapagliflozin in patients with type 2 diabetes inadequately controlled with metformin, glimepiride and dipeptidyl peptide 4 inhibitors: a 52-week prospective observational study. Diabetes Res Clin Pract. 2019, 151:65-73. 10.1016/j.diabres.2019.04.008

15. Syed SH, Gosavi S, Shami W, et al.: A review of sodium glucose co-transporter 2 inhibitors canagliflozin, dapagliflozin and empagliflozin. Cardiovasc Hematol Agents Med Chem. 2015, 13:105-112. 10.2174/1573399813666170816142351

16. Schubert A, Buchholt AT, El Khoury AC, Kamal A, Taieb V: Evaluating the costs of glycemic response with canagliflozin versus dapagliflozin and empagliflozin as add-on to metformin in patients with type 2 diabetes mellitus in the United Arab Emirates. Curr Med Res Opin. 2017, 33:1155-1163. 10.1080/03007995.2017.1310091

17. Lee PCH, Gu Y, Yeung MY, et al.: Dapagliflozin and empagliflozin ameliorate hepatic dysfunction among Chinese subjects with diabetes in part through glycemic improvement: a single-center, retrospective, observational study. Diabetes Ther. 2018, 9:285-295. 10.1007/s13300-017-0355-3

18. Rosenstock J, Seman LJ, Jelaska A, Hantel S, Pinnetti S, Hach T, Woerle HJ: Efficacy and safety of empagliflozin, a sodium glucose cotransporter 2 (SGLT2) inhibitor, as add-on to metformin in type 2 diabetes with mild hyperglycaemia. Diabetes Obes Metab. 2013, 15:1154-1160. 10.1111/dom.12185

19. Kovacs CS, Seshiah V, Swallow R, Jones R, Rattunde H, Woerle HJ, Broedl UC: Empagliflozin improves glycaemic and weight control as add-on therapy to pioglitazone or pioglitazone plus metformin in patients with type 2 diabetes: a 24-week, randomized, placebo-controlled trial. Diabetes Obes Metab. 2014, 16:147158. 10.1111/dom. 12188

20. Hadjadj S, Rosenstock J, Meinicke T, Woerle HJ, Broedl UC: Initial combination of empagliflozin and metformin in patients with type 2 diabetes. Diabetes Care. 2016, 39:1718-1728. 10.2337/dc16-0522

21. Romera I, Gomis R, Crowe S, et al.: Empagliflozin in combination with oral agents in young and overweight/obese Type 2 diabetes mellitus patients: A pooled analysis of three randomized trials. J Diabetes Complications. 2016, 30:1571-1576. 10.1016/j.jdiacomp.2016.07.016

22. Fioretto P, Giaccari A, Sesti G: Efficacy and safety of dapagliflozin, a sodium glucose cotransporter 2 (SGLT2) inhibitor, in diabetes mellitus. Cardiovasc Diabetol. 2015, 14:142. 10.1186/s12933-015-0297-X

23. Jabbour SA, Hardy E, Sugg J, Parikh S; Study 10 Group: Dapagliflozin is effective as add-on therapy to sitagliptin with or without metformin: a 24-week, multicenter, randomized, double-blind, placebocontrolled study. Diabetes Care. 2014, 37:740-750. 10.2337/dc13-0467

24. Matthaei S, Bowering K, Rohwedder K, Grohl A, Parikh S; Study 05 Group: Dapagliflozin improves glycemic control and reduces body weight as add-on therapy to metformin plus sulfonylurea: a 24-week randomized, double-blind clinical trial. Diabetes Care. 2015, 38:365-372. 10.2337/dc14-0666

25. Müller-Wieland D, Kellerer M, Cypryk K, et al.: Efficacy and safety of dapagliflozin or dapagliflozin plus saxagliptin versus glimepiride as add-on to metformin in patients with type 2 diabetes. Diabetes Obes Metab. 2018, 20:2598-2607. 10.1111/dom.13437

26. Frias JP, Gonzalez-Galvez G, Johnsson EK, Maaske J, Peters A: 261-OR: Dapagliflozin plus saxagliptin addon vs. glimepiride add-on to metformin in patients with poorly controlled type 2 diabetes. Diabetes. 2018, 67:A70. 10.2337/db18-261-OR

27. Wiviott SD, Raz I, Bonaca MP, et al.: Dapagliflozin and cardiovascular outcomes in type 2 diabetes . N Engl J Med. 2019, 380:347-357. 10.1056/NEJMoa1812389

28. Minze MG, Will KJ, Terrell BT, Black RL, Irons BK: Benefits of SGLT2 inhibitors beyond glycemic control-a focus on metabolic, cardiovascular and renal outcomes. Curr Diabetes Rev. 2018, 14:509-517. $10.2174 / 1573399813666170816142351$ 